\title{
CULTURAL TRANSMISSION OF MELODIC AND RHYTHMIC UNIVERSALS: 4 EXPERIMENTS AND A MODEL
}

\author{
TANIA DELGADO ${ }^{1}$, ANDREA RAVIGNANI $^{* 2,3,4}$, TESSA VERHOEF ${ }^{\# 5,6}$, BILL \\ THOMPSON $^{2,4}$, THOMAS GROSSI ${ }^{7}$, SIMON KIRBY $^{7}$ \\ *Corresponding Author: andrea.ravignani@gmail.com \\ \# Corresponding Author: tessauva@gmail.com \\ ${ }^{1}$ Department of Cognitive Science, University of California, San Diego, La Jolla, USA \\ ${ }^{2}$ Artificial Intelligence Lab, Vrije Universiteit Brussel, Brussels, Belgium \\ ${ }^{3}$ Research Department, Sealcentre Pieterburen, Pieterburen, Netherlands \\ ${ }^{4}$ Language and Cognition Department, Max Planck Institute for Psycholinguistics, \\ Nijmegen, Netherlands \\ ${ }^{5}$ Leiden Institute of Advanced Computer Science (LIACS), Leiden University, Leiden, \\ The Netherlands \\ ${ }^{6}$ Center for Research in Language, University of California, San Diego, La Jolla, USA \\ ${ }^{7}$ Centre for Language Evolution, School of Philosophy, Psychology and Language \\ Sciences, University of Edinburgh, Edinburgh, UK.
}

Humans are well-versed at processing sequences. In the lab, when confronted with language or other culturally-transmitted systems, humans introduce and amplify structural regularities making the systems easier to learn (Kirby, Griffiths, \& Smith, 2014; Kirby, 2017). Is the cultural emergence of fine-grained regularities a prerogative of language alone? Can cultural transmission explain universals in musical structure (Savage et al., 2015; Trehub, 2015) as it explains 'linguistic universals' (in the Greenbergian sense)? We tackled these questions in the lab and in-silico by adopting an iterated learning paradigm. Two experiments addressed the evolution of rhythmic structure (Ravignani et al., 2016; Ravignani et al., 2017), and two the evolution of melodic structure (using, among others, data from Verhoef, 2012; Verhoef et al., 2014). Depending on the experiment, participants were given a slide whistle or an electronic drum kit, and were asked to imitate a sound sequence to the best of their abilities. The output of one generation of participants became the input of the next generation. 
Across experiments we varied conditions known to affect transmission chains, such as immediate vs. delayed recall, within-participant vs. between-participant transmission, etc. We also introduce a probabilistic model for the latent structures underpinning rhythmic sequences, alongside a psychologically plausible posterior sampling algorithm (Ravignani et al., 2017). This allows us to obtain approximate structural descriptions of rhythmic patterns across conditions and generations. The initially random experimental stimuli became more musical. In particular, most regularities emerging from our transmission chains are statistical universals of world music (Savage et al., 2015; Fitch, 2017). Both whistles and drumming patterns became more compressible, measured in terms of decreasing entropy, and easier to learn. We found reuse of a small set of basic building blocks in the emerging systems, resulting in more predictable sequences of sounds (Ravignani, 2017). This corresponds with a musical universal, namely the repetition of melodic and rhythmic phrases in music. This reuse of elements is accompanied by a transition from continuous to discrete use of pitch contours (showing convergence to another universal; Ravignani \& Verhoef, 2017). Participants produced sequences containing melodic and rhythmic patterns, i.e. musical motifs. We also see a gradual increase in mirrored elements, which suggests that the emerging melodies contain arched contours (a common musical universal). In addition, drumming sequences became more isochronous (Ravignani \& Madison, 2017), and composed of few (categorically distributed) alternating inter-beat intervals, related by small integer ratios. Patterns transformed by between-participants transmission show similar properties to those emerging from within-participant transmission (i.e. self learning; Ravignani et al., 2017). Other melodic universals, such as the length of phrases, frequency intervals of melody contours, and organization of scales can also be measured in this data set and are currently being tested. Analysis of the probabilistic model supports these insights, suggesting that later generations show increased re-use of prototypical building blocks both within and across individual sequences (Ravignani et al., 2017). The emergence of musical structure via cultural transmission: (a) does not require semantics or learning language-like behaviours; (b) operates similarly across domains of human cognition; (c) explains characteristics of music appearing as statistical universals around the world (Savage et al., 2015).

\section{Acknowledgements}

This project has received funding from the European Union's Horizon 2020 research and innovation programme under the Marie Skłodowska-Curie Grant 
agreement No. 665501 with the research Foundation Flanders (FWO) (Pegasus2 Marie Curie fellowship $12 \mathrm{~N} 5517 \mathrm{~N}$ awarded to A.R.), a visiting fellowship in Language Evolution from the Max Planck Society (awarded to A.R.), and ERC Grant [283435] ABACUS (awarded to B.d.B.).

\section{References}

Fitch, W. T. (2017). Cultural evolution: Lab-cultured musical universals. Nature Human Behaviour, 1, 0018.

Kirby, S., Griffiths, T., \& Smith, K. (2014). Iterated learning and the evolution of language. Current opinion in neurobiology, 28, 108-114.

Kirby, S. (2017). Culture and biology in the origins of linguistic structure. Psychonomic bulletin \& review, 24(1), 118-137.

Ravignani, A., Delgado, T., \& Kirby, S. (2016). Musical evolution in the lab exhibits rhythmic universals. Nature Human Behaviour, 1, 0007.

Ravignani, A. (2017). Visualizing and interpreting rhythmic patterns using phase space plots. Music Perception: An Interdisciplinary Journal, 34(5), 557-568.

Ravignani, A., Thompson, B., Grossi, T., Delgado, T., \& Kirby, S. (2017). Evolving building blocks of rhythm: How human cognition creates music via cultural transmission. bioRxiv, 198390.

Ravignani, A., \& Madison, G. (2017). The paradox of isochrony in the evolution of human rhythm. Frontiers in Psychology, 8.

Ravignani, A., \& Verhoef, T. (2017). Which melodic universals emerge from repeated signaling games? (No. e3366v1). PeerJ Preprints.

Savage, P. E., Brown, S., Sakai, E., \& Currie, T. E. (2015). Statistical universals reveal the structures and functions of human music. Proceedings of the National Academy of Sciences, 112(29), 8987-8992.

Trehub, S. E. (2015). Cross-cultural convergence of musical features. Proceedings of the National Academy of Sciences, 112(29), 8809-8810.

Verhoef, T. (2012) The origins of duality of patterning in artificial whistled languages. Language and Cognition 4(4), 357-380.

Verhoef, T., Kirby, S. \& de Boer, B. (2014) Emergence of combinatorial structure and economy through iterated learning with continuous acoustic signals. Journal of Phonetics 43C, pp. 57-68 